\title{
Students in the Field: \\ Providing Physical and Psychosocial Care on the Epilepsy Monitoring Unit
}

Arthur Labatt Family School of Nursing, Western University, London, Canada

\section{Abstract}

The Integrative Practicum (IP) is the final course for students in Western's nursing program where we work alongside a registered nurse (referred to as a preceptor) and take on the full responsibilities of an independent practitioner. For my IP, I was placed on the Epilepsy Monitoring Unit (EMU) at University Hospital. During this time, one of the most critical areas of my growth was in delivering care that is sensitive to both physical and psychosocial needs. This article explores this learning experience in addition to providing a glimpse into the nature of nursing care on the EMU and with patients who have epilepsy.

The Integrative Practicum (IP) is the final course for students in Western's nursing program. In this final clinical rotation, we work alongside a registered nurse (RN) who acts as our preceptor. With their support, we take on the full schedule and patient load of a registered nurse and make the final step towards becoming independent practitioners. For my IP, I was placed on the Epilepsy Monitoring Unit (EMU) at University Hospital, one of the largest units of its kind in the province. ${ }^{1}$ Patients come to the single-room, 11-bed unit for continuous scalp electroencephalogram (EEG) monitoring to investigate seizure activity. Other patients are admitted to the unit after intracranial electrode insertion, a more invasive but highly effective method of localizing the onset of seizure activity. ${ }^{2}$ Every bed in the unit is also equipped with a video camera, microphone, and infrared light to enable visual and audio recordings of seizures.

My placement at the EMU was an interesting and fulfilling experience. I assumed the full responsibilities of a $\mathrm{RN}$ on the unit, which included universal tasks such as medication administration and unit-specific duties such as caring for patients while they had seizures. While I acquired many specific pieces of knowledge on the EMU, an overall theme in my learning experience was the importance of balancing physical and psychosocial (i.e., psychological, social, and spiritual) care in my professional practice. This was not a new lesson to me; I already knew this from the theoretical components of my education. However, applying this knowledge in practice and seeing the positive impacts of holistic, patient-centred care on real people truly brought the theory to life. To better understand how I was able to connect theory and practice during my IP, it may be beneficial to first establish some background on epilepsy itself.

Epilepsy is a general term to describe a brain disorder that causes seizures. ${ }^{3}$ When many people imagine seizures, they might imagine a person lying on the ground with full-body convulsions, which is a generalized tonicclonic seizure (GTC). However, seizures can also be as subtle as a brief moment of staring, which is called an absence seizure. The etiology of epilepsy can be traced to events such as head trauma or infection, but in $50-60 \%$ of the cases, the cause is unknown. ${ }^{4}$ Furthermore, it is important to note that not every individual who has had a seizure will be diagnosed with epilepsy. Seizures can occur as isolated incidents with physiological explanations such as electrolyte imbalance and alcohol withdrawal. Epilepsy, on the other hand, is a chronic disorder, which can be diagnosed at any point in someone's lifetime. In Canada, approximately 1 in 10 people have epilepsy; in the United States, 2.9 million people have active epilepsy (meaning they are currently on medication or experiencing seizures). ${ }^{5}$ Medication is the first line of treatment for epilepsy and approximately two thirds of people are able to control their epilepsy with it. ${ }^{6}$ However, for some individuals, seizure control may mean taking several medications and cycling through different kinds to find the right combination. If medication is still ineffective in achieving seizure control, others may need to look to more intensive treatment options such as Vagus Nerve Stimulators (VNS) or surgery.

In the EMU patients (also referred to as clients) are admitted for monitoring for a variety of reasons. Some 
patients may be admitted to try to achieve greater control through medication adjustment while others may wish to have their seizures classified. Several clients are admitted to the unit to assess their suitability for surgery, or for further investigation through intracranial electrodes. However, many-if not most-patients are admitted with the intention of obtaining more control in their lives through diagnosis, classification, and treatment. ${ }^{7}$ It is the responsibility of the health care team to assist patients in achieving their goals for their health and to help them achieve the quality of life that they desire.

Nurses have many other roles on the health care team besides a bedside caregiver, including: counselor, communicator, educator, advocate, change agent, leader, manager, and researcher. ${ }^{8}$ Due to these various roles and responsibilities, which all pertain to the necessity of providing both physical and psychosocial care, nurses must balance both medical and interpersonal skills. This is one aspect of nursing theory that translated into my practice in the EMU; however, for the purposes of this article I will focus on nurses as caregivers and counsellors.

With regards to physical care, most patients are independent; however, some may have other physical or developmental challenges that require more care. Generally, the majority of physical care occurred before, during, and after a seizure (referred to as the pre-, interand post-ictal periods). For instance, if a patient were to have a GTC seizure it would be critical to get to them quickly to maintain their safety; for example, patients could fall from their bed or otherwise injure themselves due to their lack of control over their body during a seizure. In the EMU, to help keep patients safe during this type of seizure, I would raise all of the bed's side rails for the duration of the seizure, roll the patient onto their side, and keep them in a side-lying position. These actions are to ensure they are safe on the bed, as well as to maintain an open airway and reduce the risk of aspiration, because patients may salivate and bite their tongue or cheek hard enough to bleed. Postictally, some patients may experience cognitive effects such as confusion and aggression, which could lead them to wander or attempt to pull out their EEG leads, the latter of which is a particular concern when patients have intracranial electrodes.

Another important aspect of nursing care in the EMU is intervening if patients are having too many seizures. Since patients are admitted for the purpose of recording seizure activity, their medication is often reduced if none are occurring. However, for some, this reduction may cause a multitude of seizures, which could be dangerous if they are occurring in frequent succession. In such a case, it is important for nurses to intervene; for instance, one of my patients had to receive several doses of midazolam, lorazepam, and IV anticonvulsant medications such as lacosamide and phenytoin, on top of an increased dose of their usual medications. Anticonvulsant medications in general have varying mechanisms of action such as through acting upon sodium ion channels or altering receptors for neurotransmitters ${ }^{9}$. Benzodiazepines are firstline therapies in these situations due to their fast onset, whereas additional anticonvulsants may be given intravenously to quickly increase the serum concentrations of the drug to prevent further seizure activity from occurring. ${ }^{10}$

Similarly, patients with intracranial electrodes may also require close monitoring and intervention. Since the electrodes are inserted surgically, these patients are at risk for complications such as meningitis, subdural or intracranial hematoma, and herniation. ${ }^{11}$ For instance, a patient I cared for was in the unit with intracranial electrodes for the second time; during her first visit, she had developed meningitis that was nearly fatal. As such, a key part of care is assessing for signs of infection and the stability of the patient's neurological status. Monitoring and maintaining the dressing's integrity as well as providing adequate pain management are other ongoing aspects of caring for these patients.

For psychosocial care, I found that addressing this aspect of nursing started with taking the time to simply speak with patients. By doing so, I noted a few common challenges that people shared, such as anxiety and depression which are higher in people who have epilepsy. ${ }^{12}$ Patients may also face other challenges such as feelings of invalidation, isolation, and lowered self-esteem. These issues can be due to any number of life factors that vary depending on the individual; however, I noticed that they were often related to other people's reactions to their seizures (e.g., fear, misunderstanding, discrimination, disbelief of the seizure's authenticity) or the limitations that the condition put on their life (e.g., having their license revoked-most patients on the unit could not drive). When some of these issues were brought up, I found that often the best thing I could do was listen with an open mind and validate their concerns without judgement, helping them explore their options for action if appropriate. 
By interacting with patients positively-that is, with respect and empathy-I was able to cultivate and develop stronger therapeutic relationships. This relationship is a critical aspect of nursing care as a whole, but especially so for psychosocial care. Mental health issues such as depression may be more difficult for people to open up about, and unlike physical needs, may be easier to hide. Thus, it is important to build trust and a positive relationship with a patient to make them more comfortable bringing their feelings forward. Naturally, some patients were very open and forthcoming with sharing their feelings, while others required more time to get to know me before they began to open up even about themselves.

During my IP, I experienced the impact that the workplace environment has on patient care. Through my experiences, I found that the EMU's unique layout and structure facilitated both physical and psychosocial care. It is a large room with all 11 beds within it where patients naturally socialize and often form support groups with one another. The fact that all patients are easily and quickly accessible helped improve the quality and timeliness of physical care. I found that this layout was more beneficial in providing care than the typical structure of hospital units, which consists of individual rooms that hold one, two, or four patients. I was able to experience and directly compare working in both unit formats during my IP, as the permanent unit was under renovations for the first half of my rotation, where I worked on the temporary unit that had a conventional layout instead. It would be intriguing to see more scientific research investigating the effectiveness of nursing care using this unit layout to compare against my experience.

Overall, the EMU provided a unique nursing environment that helped me cultivate my ability to provide care that is balanced between physical and psychosocial needs. I was also able to expand my practical skill set as a nurse and learn about the epilepsy population. Moving forward into my independent practice into different environments, my experience on the EMU has reinforced the necessity and positive effects that providing sensitive, holistic, and evidence-based practice have on overall patient care, healing, and satisfaction in any patient population. The relationships I have formed with patients on the unit will stay with me and motivate me to continue to strive for providing care that attends to all of my patients' health needs in order to help them achieve the highest quality of life possible.

\section{References}

1. Ministry of Health and Long-Term Care. New Epilepsy Monitoring Bed Units in Ontario [Internet] 2014 Mar 28 [cited 2015 Sept 4]. Available from: http://news.ontario.ca/mohltc/en/2014/03/new-epilepsymonitoring-unit-beds-in-ontario.html

2. Ross DA, Brunberg JA, Drury I, Henry TR. Intracerebral depth electrode monitoring in partial epilepsy: The morbidity and efficacy of placement using magnetic resonance image-guided stereotactic surgery. Neurosurgery. 1996; 39(2): 327. DOI: 10.1097/00006123199608000-00018

3. Fisher RS, Van Emde Boas W, Blume W, Elger C, Genton $P$, Lee P, Engel J. Epileptic seizures and epilepsy: Definitions proposed by the International League Against Epilepsy (ILAE) and the International Bureau for Epilepsy (IBE). Epilepsia. 2005; 46(4): 470. DOI: 10.1111/j.00139580.2005.66104.x

4. The Canadian League Against Epilepsy. General Information [Internet] [cited 2015 Mar 30]. Available from: http://claegroup.org/html/en/general_information.html

5. Centers for Disease Control and Prevention. Epilepsy Fast Facts [Internet] 2015 Mar 12 [cited 2015 Mar 30] Available from: http://www.cdc.gov/epilepsy/basics/fastfacts.htm

6. Centers for Disease Control and Prevention. Frequently Asked Questions [Internet] 2015 Jan 13[updated 2015 Mar 18 [cited 2015 Mar 30]. Available from: http://www.cdc.gov/epilepsy/basics/faq.htm\#How is epilepsy treated?

7. Critical Care Services Ontario, Epilepsy Implementation Task Force, Provincial Neurosurgery Ontario. Provincial epilepsy monitoring Unit (EMU) guidelines for Ontario. [Internet] 2014 Jan [cited 2015 Sept 4] Available from: http://epilepsyontario.org/wpcontent/uploads/2015/03/Provincial-Epilepsy-MonitoringUnit-EMU-Guidelines-for-Ontario_February-4-

2014_Final.pdf

8. Wilkinson JM, Treas LS. Fundamentals of nursing: Theory, Concepts, and Applications. Vol. 1. Philadelphia: F.A. Davis Co., 2010. 13.

9. Czapinski P, Blaszczyk B, Czuczwar SJ. Mechanisms of action of antiepileptic drugs. Curr Top Med Chem. 2005;5(1): 3. DOI: 10.2174/1568026053386962 
10. Kinney M, Craig J. Grand rounds: An update on convulsive status epilepticus. Ulster Med J. 2015; 84(2): 90-91.

11. Sperling MR. Clinical challenges in invasive monitoring in epilepsy surgery. Epilepsia. 1997; 38(4): S6-7. DOI:10.1111/j.1528-1157.1997.tb0451.x

12. Kwon OY, Park SP. Depression and anxiety in people with epilepsy. J Clin Neurol. 2014; 10(3), 175-77. DOI: 10.3988/jcn.2014.10.3.175 